function to identify the near-natives for the individual classes.

The scoring functions based on the complementarities of the interfaces regarding hydrophobicity, electrostatic potential and shape which highly rank near-natives than other models, were constructed using 73 non-redundant hetero-dimers. The dataset consists of various types of hetero-dimers, which could be classified into at most 5 clusters according to the above features used to identify the near-natives These clusters correlate with the groups based on the complex structures and the interface shapes. The largest cluster contains the hetero-dimers with globular shapes which may be stable. In some small clusters, the hetero-dimers with non-globular shapes are included. They are often transient complexes like the resent targets in the CAPRI experiments. Thus, we found that the hetero-dimers are structurally very diverse, but that they could be classified into several groups based on the above features along with the structural information, even without the knowledge about protein function

\section{P-045 分子動力学解析によるアクチンフィラメントの剛性評価}

Stiffness Evaluation of Actin Filament by Molecular Dynamics Analysis

Shinji Matsushita (1,2), Taiji Adachi (1,2), Yasuhiro Inoue (1,2), Masaki Hojo (1), and Masahiro Sokabe (3,4) (1: Dept. Mechanical Engineering and Science, Kyoto University; 2 . Computational Cell Biomechanics Team, RIKEN; 3: Dept. Physiology, Nagoya University; 4: ICORP/SORST Cell Mechanosensing, JST)

Actin cytoskeleton, particularly its dynamic reorganization, plays critical roles in a variety of mechanical cellular activities, such as migration, division and cell shape control. The major component of actin cytoskeleton, actin filament, has a double-helical structure consisting of globular actin molecules. Mechanical stretching, twisting, and bending cause structural changes in the double-helical filament, which must be a critical step to induce cytoskeletal reorganization observed in the above mentioned cellular activities. It is imperative that we need to understand the molecular and physical mechanisms how mechanical forces affect the structure of actin filament. Although past biomechanical studies have succeeded in estimating the mechanical properties of actin filament including extensional stiffness, little has been done on their molecular and physical basis. In this study, we focus on the relationship between the thermal fluctuations and mechanical properties of actin filament under different mechanical conditions. First we constructed an actin filament model consisting of 14 actin molecules in water solvent. Then, we analyzed its longitudinal and rotational Brownian motions using Molecular Dynamics simulations. We found that both the extensional and torsional stiffness of the actin filament, evaluated based on the principle of equipartition of energy, were altered by applied tensile forces. This seemed to arise from the modulations of mode and extent of thermal fluctuations of individual actin molecules by external forces.

2P-046 1 分子蛍光偏光法による古細菌型シャペロニンの構造変化解析

Single-molecule fluorescence polarization studies of the conformational change in archaeal group II chaperonin

Ryo Iizuka(1), Taro Ueno(1), Nobuhiro Morone(2), Yoshitaka Shirasaki(3), and Takash Funatsu(1). (1: Grad. Sch. of Pharm. Sci., The Univ. of Tokyo; 2: Nat'l Inst. of Neurosci. Nat'l Center of Neurol. and Psych.; 3: Kazusa DNA Res. Inst.

Group II chaperonins, found in archaea and in the eukaryotic cytosol, mediate protein folding without GroES-like cochaperonin. Structural studies suggest that the apical domains rotate $40-70^{\circ}$ in clockwise direction to seal off the central cavity upon the binding of ATP. To describe the conformational change in detail, we conducted single molecule analyses by fluorescence microscopy using the chaperonin from a hyperthermophilic archaeum, Thermococcus sp. strain KS-1 To reveal the orientation of the apical domain by analyzing that of the fluorophore, two cysteine residues were introduced at the tip of the domain, and were labeled with a bifunctional thiol-reactive rhodamine (BSR). BSR-labeled chaperonin was directly immobilized onto the coverslips treated with an $\mathrm{O}_{2}$ plasma asher. Judging from platinum rotary-shadowed electron micrograph and defocused fiuorescence images, almost all of the chaperonins are found to be in a top-view orientation. The conformational change induced by ATP was detected by the following approaches: (i) the modulation of excitation polarization to determine the in-plane angle of a BSR molecule, and (ii) the analysis of the polarization of fluorophore's emission. In both approaches, the abrupt change in the orientation of BSR was observed upon addition of ATP at $50{ }^{\circ} \mathrm{C}$. In the meeting, the details will be discussed.

\section{P-047 非平衡分子動カ学シミュレーションによる結合の自由エネルギー の順序予測}

Prediction of the order of binding free energies by non-equilibrium molecular dynamics simulations

Okimasa Okada (Medicinal Chemistry Laboratory, Mitsubishi Tanabe Pharma Corporation)

Many methods in structure-based drug design (SBDD) have been developed experimentally and theoretically. However, no standard method has been established and we still have to rely on the knowledge and experiences piled up for long years. One of the reasons is that the prediction of affinity of compounds is extremely difficult. As the affinity is related to the binding free energy dG, which is expressed by enthalpy and entropy terms, several methods to predict the $\mathrm{dG}$ have been proposed. Since these methods need huge amount of computation and the results are not always reliable due mainly to inaccurate estimation of the entropy term, we cannot routinely use them in SBDD at present. In 1997 Jarzynski proved that the exponentially weighted average work done on the system by non-equilibrium experiments is equal to dG. Steered molecular dynamics calculation (SMD), which applies external force to the system, can be used to integrate the work, where the entropy term is included. In SBDD, selection of high affinities compounds from candidates in a short time could be more useful than the time-consuming exact estimation of $\mathrm{dG}$. Therefore, we performed SMD to predict the order of the affinities with relatively small amount of computation. p38 (kinase) was used as a model protein with nine compounds, whose complex structures and dissociation constants had been published. Each compound was pulled out of the cleft by moving $20 \AA$ in 40 ps. We obtained a good correlation of the calculated work with $\mathrm{dG}$ and we think this could be a promising method.

\section{P-048 翻訳終結因子 RF3 の X 線結晶解析}

The crystal structure analysis of release factor 3 from sulfate-reducing bacterium Kiyohito Kihira (1), Shuko Numata (2), Masaya Kitamura (2), Jun Kondo (3), Shinichi Terawaki (1), Yasuhito Shomura (1), Hirofimi Komori (1), Naoki Shibata (1) and Yoshiki Higuchi. (1: Graduate School of Life Science, Univ of Hyogo; 2: Graduate School of Engeneering, Osaka City Univ; 3: Mitsubishi Tanabe Pharm. Co.)

Translation of an mRNA is terminated when a stop codon is encountered. Since tRNAs do not recognize stop codons, this event is performed by proteins called release factors. Prokaryotes have two class I release factors (RFs), RF1 and RF2, and one class II release factor, the G protein RF3. RF1 and RF2 hydrolyze and release the completed polypeptide from the peptidyl-tRNA at the ribosomal P-site in response to a stop codon. RF1 recognizes the stop codons UAA and UAG, whereas RF2 recognizes UAA and UGA. RF3 binds to the ribosome to promote rapid dissociation of RF1 or RF2 from the A-site in a GTP-dependent manner. We have studied the structure-function relationship of the RF3 found in sulfate-reducing bacterium, Desulfovibrio vulgaris Miyazaki F. Here we present the high resolution crystal structure of RF3-GDP complex. The polypeptide chain of RF3 in complex with GDP is folded into three distinct domains. Domain $I$ is composed of a classic GTPase domain (G domain) and an 'EF-G-like' subdomain. Domain II forms a $\beta$-barrel structure as observed in EF-Tu, eRF3 and EF-G. Domain III is composed of a central $\beta$-barrel flanked by two $\alpha$-helices. Structural comparison with $E$. coli RF3 in the GDP-bound form as well as EF-G-2 and EF-Tu in the GTP-bound forms is also described.

\section{P-049 金属蛋白質の QM/MM 計算における遠距離静電相互作用の影響}

Effects of long-range electrostatic interactions in QM/MM calculations onto active sites of metal-binding enzymes

JiYoung Kang (1), Takehiro Ohta (1), Yohsuke Hagiwara (1), Keigo Nishikawa (2), Tetsunori Yamamoto (2), Hidemi Nagao (2), and Masaru Tateno (1) (1: Center for Computational Sciences, University of Tsukuba, 2: Graduate School of Natural Science and Technology, Kanazawa University)

Ab initio electronic structure calculations are indispensable to elucidate functional mechanisms of metalloenzymes, such as electron/proton transfer. Extracted model structures of active sites have been often exploited so far; but surrounding protein structures are suggested to actually modulate the mechanisms. To elucidate this, joint schemes of quantum mechanics (QM) for metal binding sites and molecular mechanics (MM) for surrounding QM regions are utilized in a modeled system in this study.

Using azurin as an instance of blue $\mathrm{Cu}$ enzymes, we investigated the following two types of QM/MM schemes with use of our new interface program to connect QM (gamess) and MM (amber) engines (see other posters presented by us): In a "subtracted" scheme, electrostatic interactions between QM/MM regions are truncated in QM calculations; on the other hand, in an "additive" scheme, long-range electrostatic interactions within a cutoff distance from QM atoms are introduced into one-electron integration terms of the QM Hamiltonian.

As a result, we have found significant differences of geometrical and electronic structures optimized using two QM/MM joint schemes: For bond lengths between $\mathrm{Cu}$ and coordinated atoms, which are sensitive to redox potentials of azurin, polarized bonds, such as $\mathrm{C}=\mathrm{O} \cdots \mathrm{Cu}$ and $\mathrm{S} \cdot \cdots \mathrm{Cu}$, are indicated to be definitely influenced by long-range electrostatic interactions between QM/MM regions. Thus, additive schemes are required to obtain accurate descriptions of mechanisms related to functions of metalloenzymes.

\section{P-050 水晶体特異的蛋白質 Filensin と Phakininがつくる中間径フィラ メントの形態解析}

Morphological analysis of the intermediate filaments formed by lens-specific proteins filensin and phakinin in vitro

Roichi Nakamuta (1), Hiroyuki Ainobu (2), Masaya Wada (2), Taketsune Matsuzaki (1), Yushi Oishi (2), Mikako Oka (3), Makoto Takehana (3), Yozo Takasaki (1), Shoji Ando (1).(1.Dept Biomolecular Sciences, Fac Medicine, Saga Univ; 2:Fac Science and Engineering, Saga
Univ; 3 Fac Pharmacy, Keio Univ)

Lens fiber cells of the eye expresses two specific cytoskeletal proteins known as phakinin and filensin. These two proteins constitute unique lens filaments, called beaded filaments (BFs), which are assumed to function in the maintenance of lens transparency. Native BFs have globular particles with a diameter of $12-15 \mathrm{~nm}$ that decorate a $6-8 \mathrm{~nm}$ filament backbone at regular intervals. It is still debatable whether the globular particles of BFs are constructed by the C-terminal tail domain of filensin or by a third component such as crystallins. In addition, although the tail domain of filensin is processed during aging and cataractogenesis, it is not well understood to what extent the processing affects the structure and the function of BFs. In this study, we prepared recombinant proteins of rat phakinin (49 kDa), filensin ( $94 \mathrm{kDa}$ ), filensin mutants ( 50 and $38 \mathrm{kDa}$ ) in which the tail domain is stepwise deleted, and examined their competences to 